\title{
Automatic Recognition of Road Sign Passo-Carrabile
}

\author{
Luca Lombardi, Roberto Marmo, and Andrea Toccalini \\ Dipartimento di Informatica e Sistemistica, University of Pavia, 27100 Pavia, Italy \\ \{luca. lombardi, roberto.marmo\}@unipv.it \\ http://vision.unipv.it
}

\begin{abstract}
This paper describes a method to detect and identify the typical italian road sign passo-carrabile. The system first determines the region of interest within the image, using color segmentation, then the signal of no waiting is identified using shape and color information, and finally the text label passo-carrabile is recognised with a state diagram and a set of specific tests on the shape of the words. The obtained results show the feasibility of the system.
\end{abstract}

\section{Introduction}

Discriminations between road signs can be performed mainly by its colors, which are chosen to identify the sign easily and differentiate it from the background. According to this, the first analysis consists in color segmentation. One problem is the high sensibility of colors to weather changes, so this first elaboration is applied keeping a high tolerance. The italian road sign Passo-carrabile can have different dimensions, but the standard model (Fig. 1) is $24 \mathrm{~cm}$ wide and $43 \mathrm{~cm}$ long, with a no waiting signal of $10 \mathrm{~cm}$ radius; this sign is painted only with red, blue, white and black colors 9. This specific sign gives the order to keep clear a transit zone, it forbids parking in a lateral area of the street located into town, so other objects with similar colors can make more difficult the recognition.

It is important to examine also the text label passo-carrabile as an additional test and to differentiate this specific sign among all other prohibition signs.

The algorithm created to find the road sign is defined on three levels. First, the program performs a rapid analysis of the whole image, and selects the area that will be analyzed more accurately, according to the distribution of the sign colors, next levels work on the selected region; in case of negative response the control turns back to the first level, and the research continues on the image. If the previous constrains are satisfied, we use the Hough Transform to find red and blue circles, which correspond to disks of the prohibition signal: if circles are detected and they have a common center, we use specific measurements to estimate the real dimensions of the sign. If obtained results are admittable, the program continues with more detailed controls, so we apply a pattern matching of the prohibition signal with a model, and the analysis of the text label 'passo carrabile' with a set of specific tests. 


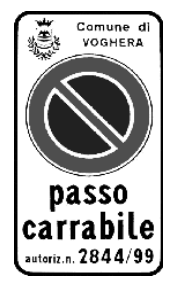

Fig. 1. The road sign passo-carrabile

\section{Color Segmentation}

Color segmentation is the most critical point of the algorithm: it is the first operation executed, so it is very important to keep high tolerance, to avoid loosing useful informations.

The color space RGB because is the typical model used by digital cameras, so we do not need any transformation. One problem working with the RGB rapresentation is the high sensibility to lighting conditions, so we study relations between color components 14510. Converting the RGB space to HSV (Hue, Saturation, Value) allows to gain a better control on chromatic variations of each hue (it is a separated component), but the computational cost is prohibitive, because the space transformation is nonlinear. HSV space is very tipic 367811 , but some studies shows that Hue component changes considerably according to distance, brightness and sign age. Thus we decided to use the RGB model to reduce execution time, also because we analyze only two colors (red and blue) and these are represented by two separately channels in RGB space; then white color can be easily obtained putting together the three components.

Now we extract a region of the image with enough quantity of these colors. Three color image histograms (Fig. 2) are analyzed to find a point with a sufficient number of colored pixels, of the 3 interested colors, on the same row and also on the same column [12].

\section{Analysis of the Region of Interest}

The algorithm for circles detection works on binary images, the blue and red masks of the inspected image area. We have refined the colors segmentation, adjusted on the mean brightness of the analyzed region. A gaussian filter (mean value among adjacent pixels) removes noise, also deleting a lot of isolated pixels which would increase noise for Hough method.

Now the edges of the two binary images are extracted: for this operation we use the isotropic operator, which consists in the convolution of the image with $3 \times 3$ masks.

Hough transform then is performed to locate red and blue circles. We do not know a priori the radius of these circles, so we search in a three-dimensional parameter space to find the three values $\left(x_{c}, y_{c}, r\right)$ corresponding to circumference. We apply the Hough method either on red and blue circles; it allows to 


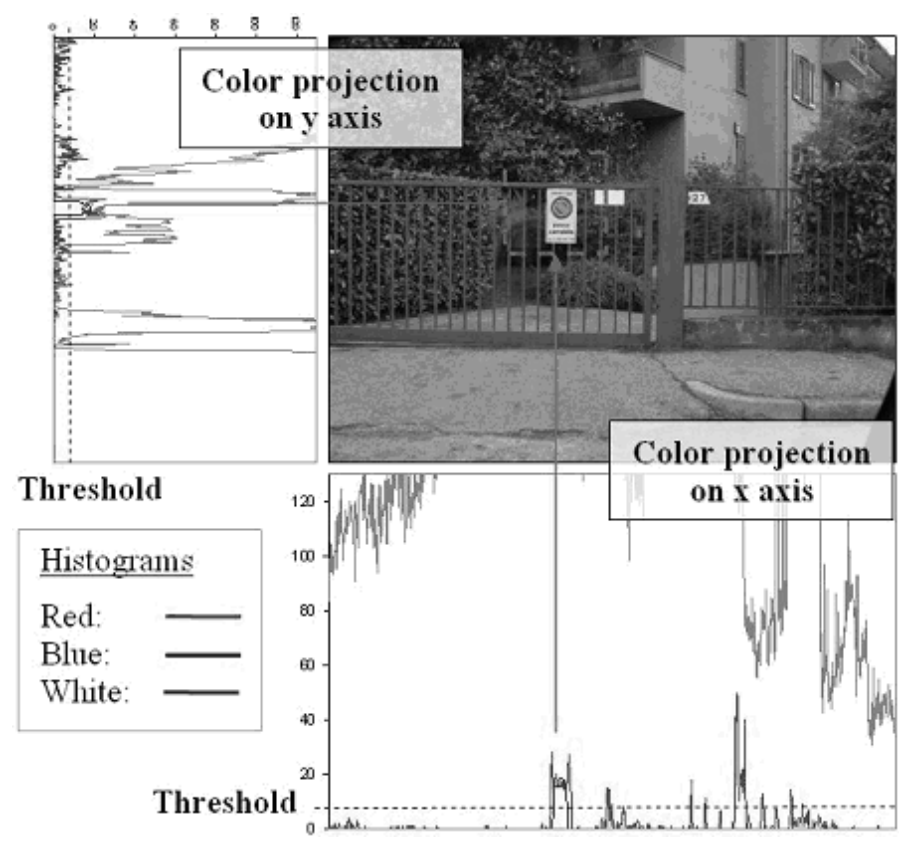

Fig. 2. Extraction of the interesting image area using histograms of colors red, blue and white

detect the center of the signal with good assurance and approximate sign dimensions. We needed to perform some optimizations to get this algorithm as lighter as possible. Now, using these results we want to find exactly the position and dimensions of the signal painted on the road sign. The idea consists in performing 8 measurements on the signal, obtaining a good strength even if we have occlusions, and also verifying the results accuracy in most common cases.

Starting from the center found with Hough, we execute 4 scannings (right, left, up and down directions) and we store positions of color changes, from blue to red and then to white. So we can identify the dimensions of the three different regions within the signal, from these values we can easily detect the radius of the blue and the red rings and obtain the radius of the signal (Fig. 33).

At this point we know exactly the position and the dimension of the signal, so we can apply a pattern matching $[6,10]$ with a sample image [11] (Fig. [3).

We modify the image which contains the sign to verify, to improve the results of this technique: a coloration is applied before pattern matching, every colored pixel is altered to its pure tonality (for example: $R=255, G=0, B=0$ for every red pixel). By this way we have good results even if the road sign is dirty or affected by bad lighting.

Finally, we apply a further control on the no-waiting signal, to identify and exclude the no-stopping signs: these are very similar signs, the main difference is a double red strip instead of a single one (Fig. 4). These signs are able to pass the pattern maching test with positive result. The approach consists in counting 


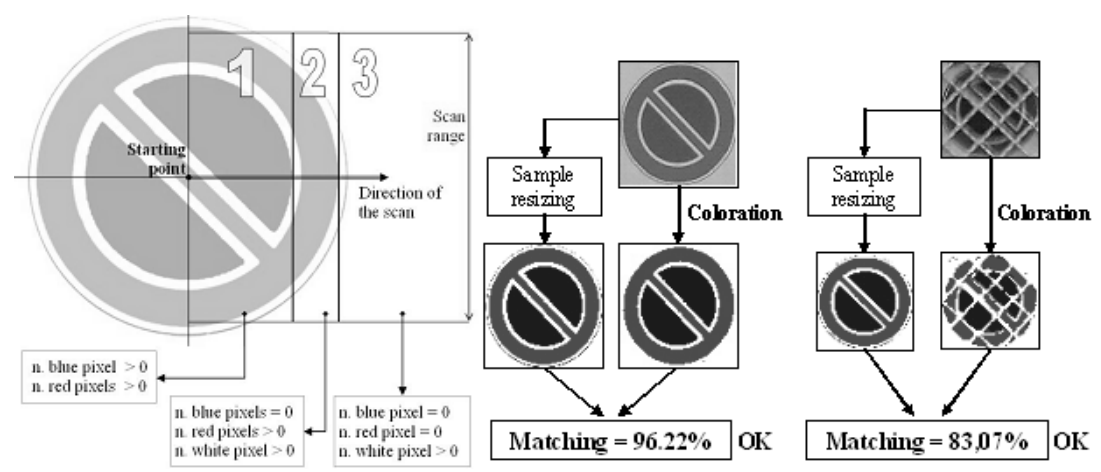

Fig. 3. Extraction of sign dimensions: on the left horizontal scan to right direction, on the right examples of pattern matching

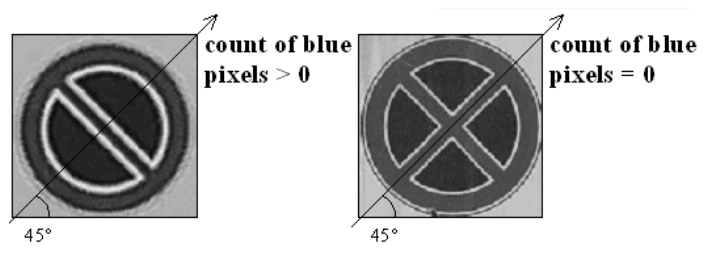

Fig. 4. No-waiting (left) and no-stopping (right) signs

the number of blue pixels on the diagonal placed at +45 . When the signal has a double red strip we obtain that this number is equal to zero, in the other case the number of blue pixels always be major then zero. This procedure always given the right result.

\section{Text Extraction}

The text label passo-carrabile is located in a specific region, under the signal of no-waiting on the bottom of the sign. It is possible to identify characters using an O.C.R. method, but in this case we realized that it was sufficient to apply some controls on writing characteristics, there is no need to recognize each letter: thus, we can semplify and speed up the execution. Moreover, if the road sign is placed far enough from the acquisition source, the writing is displayed with very low resolution and so it is impossible to recognize exactly each character.

\subsection{Pre-elaboration}

We need to correct brightness and contrast of this zone, to get a better image with a clear and well-defined writing (Fig. 5). Then the image is binaryzed according to a threshold value, to simplyfy next operations like characters extraction and testing. We use dynamic parameters to perform these adjustments to the image: they are related to the mean brightness of the region analyzed. 


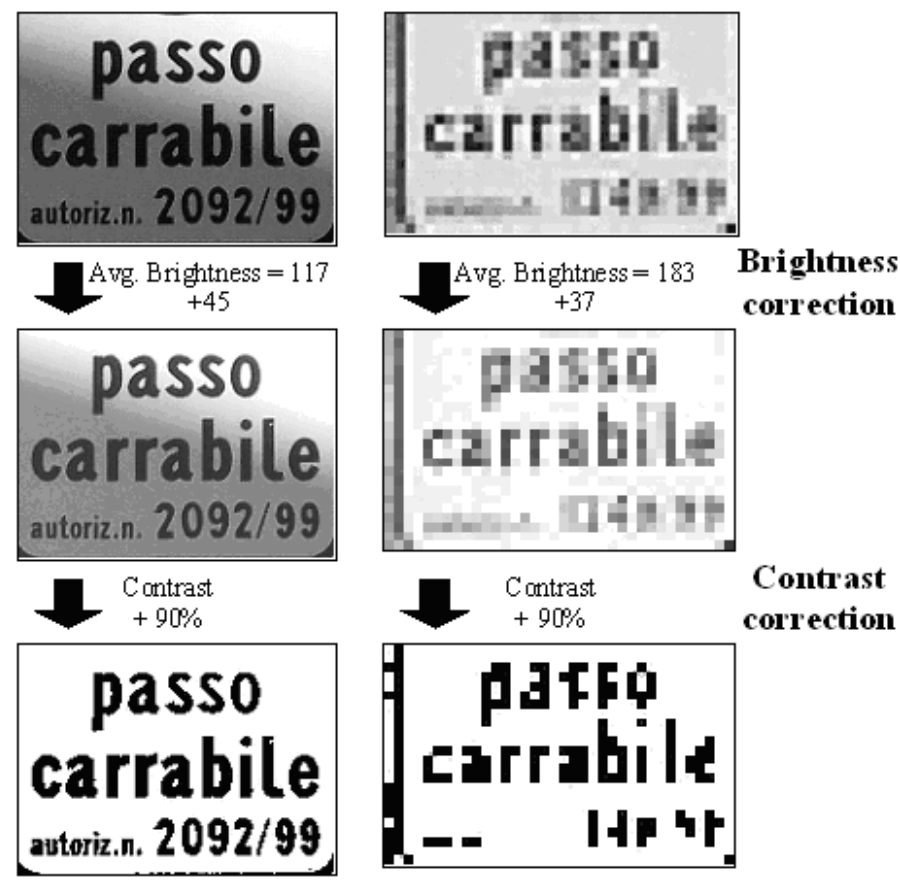

Fig. 5. Brightness and contrast adjustment

\subsection{Words Extraction}

To identify the exact dimensions and positions of the two words, passo and carrabile, we execute a vertical scansion of the binary image, analyzing the black projection on the y axis [2. Working with a finite state machine we can detect both position and height of the two words (which are always one above the other), as we examine the percentage of black pixels located on each row. Sometime the sign is placed beyond a gate of the house and we obtain a noise caused by gate. We can find out this constant error performing the previous scanning, and we achieve good results even if the sign is corrupt in this way.

We can identify the error $\epsilon$ using informations from the first derivative of y projection, by the incremental ratio: $y^{\prime}(i)=y(i+1)-y(i)$ where $y$ vector contains, for each element $i$, the count of black pixels situated on the image row $i$; vector $y^{\prime}$ represents its derivative, i.e. the variation of of black pixels between two adjacent rows. Examining the vector $y^{\prime}$, we can note positive peaks corresponding to upper boundaries of words, and negative peaks on words ends: our technique consists in detecting these peaks (so locating where the words are) and extract e as the minimum value $y(i)$ among all rows $i$, outside these words, where $y^{\prime}(i)=0$. Briefly, we calculate the fixed error as the minimum number of black pixels located on white rows; we also assume that this noise vertically involves all the image (Fig. 6). So, we obtain two images which fits exactly the two words we have to examine. 


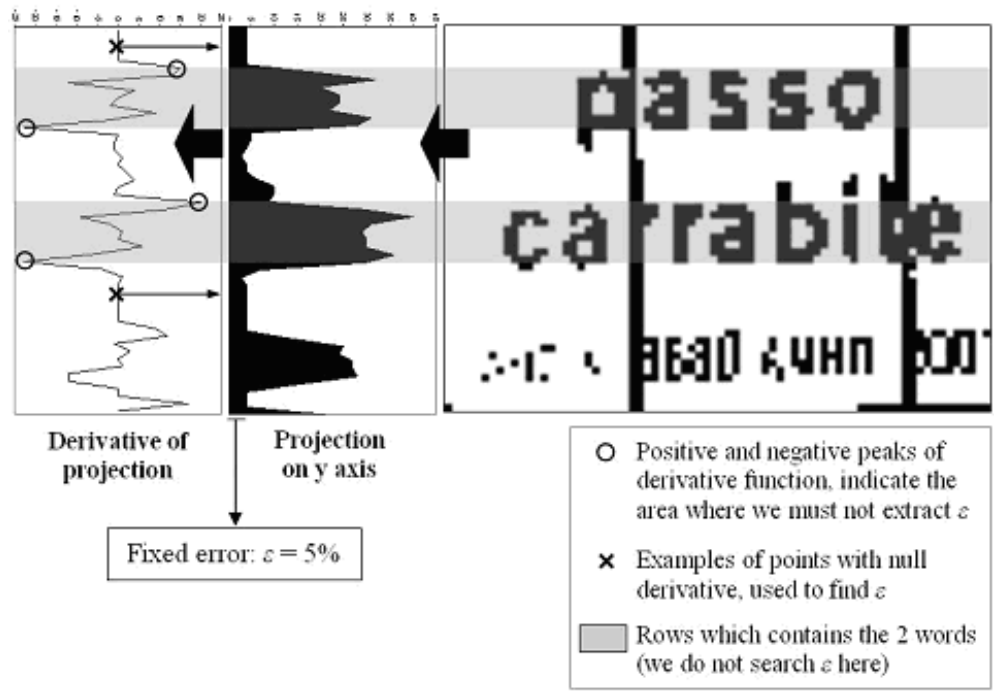

Fig. 6. Extraction of a fixed noise, using the derivative of projection on y axis
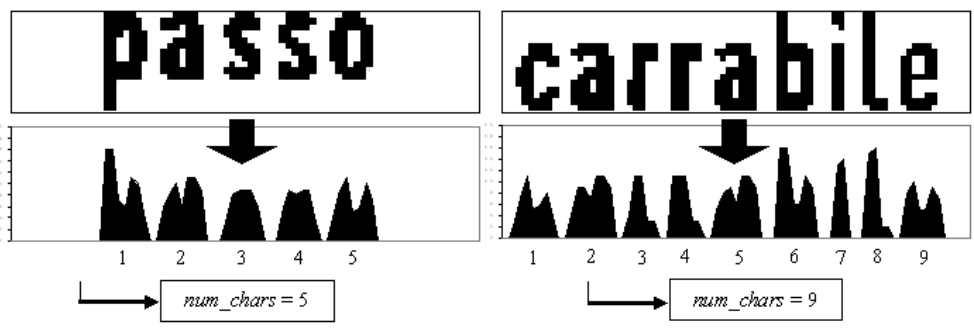

Fig. 7. Projection of black pixels on $\mathrm{x}$ axis

\subsection{Characters Extraction}

Using horizontal scans on the two words separately we can extract the number of characters, their dimensions and width analyzing the count of black pixels on each column of the image (Fig. 7). We use again a state diagram, where the transition from states 'character' and 'space' is defined by the percentage of black pixels on each column. We refine this technique, since we often have low resolution or noise on the text: for example it is useful to verify the width of each character in comparison with the median width of detected characters, realizing if characters are too large or too tight.

\subsection{Final Tests}

We perform some specific tests, each one allows only a little tolerance of dissimilarity from the model of a standard sign. The identification of the label is 
verified succesfully if sufficient number of tests have positive results (not necessarily everyone), so we can also remove a high number of false positives (thanks to the low tolerance of each test). Briefly, this method allows to recognize the label passo-carrabile if it has enough common features with a sample image and using this tests:

1. analogy of heights of the 2 words;

2. word 'carrabile' larger then word 'passo';

3. width of the 2 words proportionate;

4. all characters of 'passo' with adequate width;

5. all characters of 'carrabile' with adequate width;

6. count of characters from 'passo' equal to 5;

7. count of characters from 'carrabile' equal to 9 .

Each test has a different weight, relative to its importance for the recognition; if we still have an uncertain result after these 7 tests, we apply other 6 verifications:

8. width of word 'passo' proportional to width of the sign;

9. height of word 'passo' proportional to height of the sign;

10. matching between the 2 characters 's' from the word 'passo';

11. matching between the 2 characters ' $r$ ' from the word 'carrabile';

12. matching between the word 'passo' and a sample image;

13. matching between the word 'carrabile' and a sample image;

Table 1. Confusion matrix: $c_{i j}$ coefficient at row $i$ and column $j$ represents the percentage of sign of class $i$ identified as sign of class $J$

\begin{tabular}{|l|l|l|}
\hline \multirow{2}{*}{ predicted classification } & real classification \\
\cline { 2 - 3 } & positive & negative \\
\hline positive & 204 & 6 \\
\hline negative & 0 & 210 \\
\hline
\end{tabular}
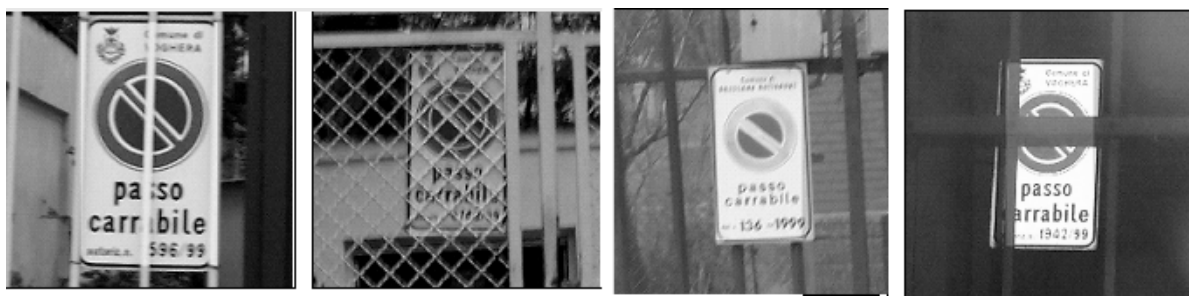

Fig. 8. Examples of right results 


\section{Results}

Results produced by the developed system are satisfying, as we recognize correctly the signs of passo-carrabile under different lightness conditions and also with high noise. We have tested the program on a set composed of 420 images, with a resolution of $1024 \times 768$, on a computer with a Intel Pentium 4 processor at $3 \mathrm{GHz}$ and $512 \mathrm{MB}$ of RAM DDR memory at $400 \mathrm{MHz}$. Concerning the computational times, the mean execution time is $74 \mathrm{~ms}$ and the frame per second is 13.5.

The confusion matrix (Tab. 1) shows that system accuracy is equal to $98.6 \%$. As we can see, the program works very good with false positives: thanks to the low tolerance of last tests on the label passo-carrabile we do not have any false positive. The system is very strong against noises and occlusions of the signal, as we recognize the road sign even in very prohibitive circumstances (Fig. 8). The few failures are caused by too heavy noise on the writing or excessive faded colors.

The testing has been performed acquiring images at different distances from the sign, from 1 meter to more then 10 meters far. Sometimes the road sign is placed far from the acquisition source, so we need high image resolution to text recognition. Using a resolution of $1024 \times 768$ we experienced good results and text can be correctly detected until a distance of 11.5 meters, characters are 2 pixels wide and 3 pixels high and the sign radius measures only 11 pixels.

\section{References}

1. Escalera, A., Moreno, Salichs, Armigol.: Road Traffic Sign Detection and Classification. IEEE Transactions on Industrial Electronics 44 (1997) 848-859

2. Miura, J., Kanda T., Shirail Y.: An Active Vision System for Real-Time Traffic Sign Recognition. Proc. of IEEE Conference on Intelligent Transportation System (2000) $52-57$

3. Escalera, A., Armingol, J., Mata, M.: Traffic sign recognition and analysis for intelligent vehicles. Image and Vision Computing 11 (2003) 247-258

4. Zadeh, M., Kasvand, T., Suen, C. Y.: Localization and Recognition of Traffic Signs for Automated Vehicle Control Systems. Conference on Intelligent Transportation Systems, Pittsburgh, PA, (1998) 272-282

5. Bnallal, M., Meunier, J.: Real-time color segmentation of road signs. IEEE Canadian Conference on Electrical and Computer Engineering, Montral, Qubec, Canada, (2003) 1823-1826

6. Vitabile, S., Pilato, G., Pollaccia, G., Corbello, F.: Road Signs Recognition Using a Dynamic Pixel Aggregation Technique in the HSV Color Space. Proceedings of 11 International Conference on Image Analysis and Processing, Palermo, Italy, (2001) 572-577

7. Gavrila, D. M.: Traffic Sign Recognition Revisited. Proceedings of the 21st DAGM Symposium fr Mustererkennung, Springer Verlag, Bonn, Germany, (1999) 86-93

8. Liu, H. X., Ran, B.: Vision-Based Stop Sign Detection and Recognition System for Intelligent Vehicles. Transportation Research Record 1748 (2001) 161-166

9. Italian Highway Code, http://digilander.libero.it/ordinanze/cds/codice.htm 
10. Sekanina, L., Torresen, J.: Detection of Norwegian Speed Limit Signs. Proceedings of 16th European Simulation Multiconference, Darmstadt, Germany, (2002) 337340

11. Vitabile, S., Gentile, A., Dammone, G., Sorbello, F.: MLP Neural Network Implementation on a SIMD Architecture. Lecture Notes in Computer Science 2486, Springer-Verlag, (2002) 99-108

12. Piccioli, G., DeMicheli, E., Campani, M.: A Robust Method for Road Sign Detection and Recognition. Proceedings of Third European Conference on Computer Vision, (1994) 495-500 